\title{
Radiothérapie : les leçons à tirer des accidents d'Épinal et de Toulouse
}

\author{
M. BOURGUIGNON ${ }^{1}$, J.M. SIMON ${ }^{2}$, D. PEIFFERT $^{3}$, D. KREMBEL ${ }^{1}$
}

(Manuscrit reçu 16 juin 2009, accepté le 15 octobre 2009)

RÉSUMÉ Les accidents de radiothérapie d'Épinal et de Toulouse, identifiés respectivement en 2006 et 2007, ont concernés un très grand nombre de patients. La sur-irradiation des organes cibles lors de la radiothérapie a entrainé des effets déterministes graves. Un retour d'expérience a été effectué et a indiqué que les erreurs qui conduisent aux événements graves résultent de défaillances humains et organisationnelles. C'est dans ce domaine du facteur organisationnel et humain que doivent porter les efforts pour prévenir la survenue de nouveaux accidents.

ABSTRACT Radiation therapy: the lessons from Épinal and Toulouse accidents.

The radiation therapy accidents of Epinal and Toulouse which were identified in 2006 and 2007 respectively concerned a large number of patients. The overexposure of the target organs during radiation therapy induced severe deterministic effects. A return of experience has been made. It showed that the mistakes which occurred resulted from organisational and human failures. Thus, it is in this domain that efforts must be carried out to prevent new accidents.

Keywords: radiation protection / radiological accidents / radiotherapy / Épinal

\section{Introduction}

L'accident de radiothérapie d'Épinal est le plus important de ces dernières années dans le monde. Il convient donc d'analyser en profondeur les mécanismes qui ont conduit à la sur-irradiation de plus de 5000 patients pendant près de 20 ans afin d'en tirer des leçons à des fins de prévention de nouveaux accidents.

Les leçons à tirer de cet accident peuvent être complétées par les observations résultant des autres événements de radiothérapie qui ont été déclarés à l'ASN et par celles que l'ASN a pu effectuer lors de ses inspections. Dans la très grande majorité des cas, les accidents et événements indésirables sont liés au facteur organisationnel et humain, les humains pouvant être défaillants et faire des erreurs qui ne sont pas rattrapées quand le contexte organisationnel est lui-même défaillant.

Autorité de sûreté nucléaire, 6 place du colonel Bourgoin, 75572 Paris Cedex 12, France.

Service de radiothérapie, CHU Pitié-Salpêtrière, 47 boulevard de l'Hôpital, 75013 Paris, France.

Service de radiothérapie, CRLCC Alexis Vautrin, 6 avenue Bourgogne Brabois, 54500 Vandoeuvre les Nancy, France. 
En identifiant les erreurs, il ne s'agit pas de chercher et de pointer du doigt un bouc émissaire. Ce document n'a pas non plus pour objet de déterminer des responsabilités qui sont du ressort de la justice, l'accident d'Épinal comme d'autres événements faisant l'objet de poursuites judiciaires. Mais il ne s'agit pas non plus d'ignorer les erreurs commises : une erreur n'est pas une faute mais la répétition d'une erreur, dès lors qu'elle a été identifiée, devient une faute. Le retour d'expérience est l'étape nécessaire pour prévenir la survenue de nouveaux accidents (Peiffert et al., 2007 ; Marchesi et al., 2009).

La liste de toutes les actions de l'ASN entreprises en ce qui concerne la radiothérapie et en particulier à la suite de l'ensemble des événements déclarés à l'ASN figure en annexe.

L'accident d'Épinal et les autres événements survenus en radiothérapie ne doivent pas faire perdre de vue que la radiothérapie française est de grande qualité puisque près de 200000 patients sont traités chaque année avec de très bons résultats et environ $50 \%$ de guérisons. Par ailleurs, le nombre des événements indésirables déclarés à l'ASN reste faible, environ 200 événements par an, en grande majorité de niveau 0 et 1 (sans conséquences pour les patients) de l'échelle ASN-SFRO et environ 5 événements de niveau 2 (avec conséquences pour les patients), statistique prenant en compte $30 \%$ des centres qui ont déclaré leurs événements indésirables en 2008.

\section{Les événements d'Épinal}

Cinq événements différents sont arrivés à Épinal entre 1987 et 2006. À la suite de la déclaration en 2006 par l'agence régionale d'hospitalisation (ARH) Lorraine du plus récent d'entre eux, ils ont été identifiés rétrospectivement après la déclaration par des patients de symptomatologies qui n'entraient pas dans le cadre de l'accident déjà identifié. C'est ainsi que les 4 autres événements ont été mis en évidence en remontant dans le temps. La chronologie et les caractéristiques de ces événements sont présentées dans le tableau ci-après.

\begin{tabular}{cccccc}
\hline Épinal & Date & Erreur & Cancer & Nombre de patients & Dose \\
\hline I & mai 2004-août 2005 & logicielle & prostate & 24 & +20 à $30 \%$ \\
II & oct. 2000-oct. 2006 & imagerie portale & prostate & 411 & +8 à $10 \%$ \\
& & & & 300 & $+7,1 \%$ \\
III & $1987-2000$ & calcul & tous sauf & 3600 & $+5,5 \%$ \\
& & & sein & 1100 & $+3 \%$ \\
IV & juillet 1993 & calcul coins & sein & 8 & +20 à $68 \%$ \\
V & 1999 & & sein & $9 / 37$ & Cœur \\
\hline
\end{tabular}




\section{Premier accident d'Épinal : mai 2004 - août 2005}

C'est en 2004 qu'une nouvelle technique de modulation de l'intensité des faisceaux de radiothérapie est mise en route au centre hospitalier d'Épinal pour le traitement des cancers de la prostate. Elle est justifiée pour mieux cibler la tumeur, améliorer la protection des tissus sains et ainsi délivrer une dose curative plus élevée.

L'appareillage disponible à Épinal était susceptible de réaliser ces traitements depuis quelques années. Leur mise en route a fait l'objet d'une initiative individuelle et non d'un projet d'équipe structuré. Du fait d'une formation insuffisante et entachée d'erreur d'un groupe de manipulateurs, un surdosage a été rendu possible. À noter la formation correcte un mois plus tard d'un second groupe de manipulateurs.

Sur le plan technique, l'erreur a consisté en la planification d'irradiations avec la mise en place dynamique de collimateurs en forme de coins, suivie de la réalisation effective des irradiations en mode statique. Il en est résulté une délivrance de doses plus élevées dans un champ d'irradiation plus large que prévu. Ces surdosages sont passés inaperçus du fait de l'absence de contrôle indépendant de la dose (logiciel maison «bricolé », système non intégré de radiothérapie) et de l'absence de dosimétrie in vivo. La surdose de 20 à $30 \%$ a concerné 24 patients parmi les 99 qui ont été traités par cette technique.

Les premiers signes cliniques ont été signalés en janvier 2005, mais sans doute minimisés par les médecins référents et les radiothérapeutes. L'alerte a finalement été donnée par un gastroentérologue de Nancy qui a observé une rectite radique chez plusieurs patients traités à Épinal. Il a fallu attendre août 2005 pour voir l'arrêt de l'utilisation de la méthode.

L'accident a été déclaré à la direction de l'hôpital en septembre 2005, notification transmise à l'ARH. Cependant l'alerte nationale n'a été effective qu'en juillet 2006.

Les leçons à tirer:

- favoriser les équipements de radiothérapie totalement intégrés, de la planification jusqu'à la réalisation du traitement, pour bénéficier de l'assurance qualité logicielle des constructeurs et éviter les anomalies possibles aux interfaces informatiques ;

- ne pas mettre en route de nouvelles méthodologies de radiothérapie sans projet de groupe anticipé, muri, préparé, sans l'écriture de procédures, sans entraînement spécifique, sans vérification de la qualité de la chaîne de 
traitement en particulier tant que les équipements ne sont pas totalement intégrés, etc.

- la formation des manipulateurs et toute délégation d'utilisation à leur niveau sont à encadrer par des procédures et une vérification de la bonne acquisition des formations est à effectuer ;

- le suivi des patients doit être rigoureux de la part des radiothérapeutes comme de celle des médecins référents avec une attention particulière à porter aux symptômes susceptibles de générer une alerte ;

- la réglementation exige que les notices d'utilisation des appareillages soient en français. Si les notices en anglais ne posaient pas de problème au physicien d'Épinal dont l'anglais est la langue maternelle, ce n'était pas le cas pour les manipulateurs. C'est une question difficile car une bonne documentation en anglais vaut mieux qu'une mauvaise traduction ;

- la déclaration des événements, obligatoire, doit être réalisée rapidement tant au niveau national que local, afin de mettre en place une prise en charge de l'envergure voulue ;

- l'information des patients est également obligatoire pour tout événement indésirable. Elle doit être réalisée rapidement par les professionnels afin que les patients ne prennent pas connaissance de l'événement par la presse.

\section{Deuxième accident d'Épinal : octobre 2000 - octobre 2006}

Lors de la phase d'instruction du premier accident, il est apparu que des patients qui présentaient des symptômes analogues à ceux présentés par les patients du $1^{\text {er }}$ accident n'avaient pas été traités par la technique de modulation d'intensité. Après examen des dossiers, il est apparu que ces patients avaient eu une imagerie portale à chaque séance de radiothérapie, apportant un surdosage de 8 à $10 \%$ non pris en compte.

L'imagerie portale consiste en la réalisation d'une imagerie avec l'appareil de radiothérapie. Elle est occasionnellement effectuée dans le cadre du contrôle de qualité du repositionnement du patient. La dosimétrie surajoutée, qui est négligeable en cas d'utilisation occasionnelle, ne l'est plus lors d'une utilisation quotidienne de l'imagerie portale.

In fine, 411 patients traités pour un cancer de la prostate ont été concernés par cette sur irradiation entre octobre 2000 et octobre 2006. Ces patients étaient peu ou mal suivis et la détection des complications non effectuée.

\section{Les leçons à tirer :}

- le suivi des patients et une attention particulière à porter aux symptômes susceptibles de générer une alerte sont à nouveau mis en évidence ; 
- l'utilisation erronée d'une technique (ici l'imagerie portale) doit être évitée par la mise en place de procédures détaillées pour tous les aspects techniques. Le guide des procédures de radiothérapie réalisé par les professionnels (SFRO) est une référence et doit être largement utilisé.

\section{Troisième accident d'Épinal : 1987 - 2000}

À la suite de la découverte des 2 premiers accidents de radiothérapie, un numéro vert a été mis à disposition des patients et une mission de suivi a été mise en place par le ministère de la Santé et assurée en pratique par le Dr Jean-Marc Simon, radiothérapeute, avec le soutien du Pr Peiffert, d'Alain Noël et des équipes de radiothérapie et de radiophysique du centre Alexis Vautrin de Nancy.

C'est ainsi que, lors de l'analyse en juillet 2007 du dossier d'un patient traité en juillet 2000, a été mise en évidence la modification sans raison apparente des modalités d'un traitement réalisé en 2000 (en pratique, modification du nombre d'unités moniteurs délivrées par séance en cours de traitement sans raison apparente). Après analyse par l'équipe de physique de Nancy, une erreur dans la formule de calcul de la dose a été mise en évidence. C'est cette erreur générique qui a été corrigée à partir de juillet 2000. Cette erreur n'aurait sans doute jamais été identifiée si le paramétrage de la radiothérapie de ce patient n'avait pas été modifié en cours de traitement.

Cette erreur remonte à 1987 et à un changement de technique concernant la calibration des faisceaux de radiothérapie. Une modification de formule mathématique pour le calcul de la dose était nécessaire lors du passage d'une méthode DSP (distance source-peau) à une methode DSA (distance source-axe c'est-à-dire source-centre de la tumeur). La programmation informatique «maison » a été erronée.

4912 patients ont été concernés par ce surdosage. Tous les cancers sauf les cancers du sein (2 700 patientes) ont été concernés par cette erreur. Comme elle dépend de l'énergie du faisceau de rayons X, l'intensité du surdosage dépend de la machine sur laquelle ont été effectuées les traitements.

C'est ainsi que :

- 312 patients traités entre 1999 et 2000 ont eu un surdosage de 7,1\% ;

- 3600 patients traités entre 1987 et 1999 ont eu un surdosage de 5,5\% ;

- 1100 patients traités entre 1993 et 2000 ont eu un surdosage de $3 \%$.

Seuls les patients ayant eu un surdosage de 5,5\% et plus présentent des séquelles. 
Il faut noter qu'une dosimétrie in vivo avait été utilisée en 1995 pour vérifier que la dose reçue était bien identique à la dose calculée. Les valeurs mesurées étant différentes des valeurs calculées, il avait été conclu qu'il s'agissait d'un problème de paramétrage du dosimètre. Une correction empirique avait alors été mise en place pour la dosimétrie in vivo pour que ses résultats coïncident à peu prés avec ceux du calcul. Alors que l'erreur de calcul aurait pu être corrigée en 1995, elle ne l'a été qu'en 2000, et sans laisser de trace autre que celle du changement des paramètres de traitement d'un patient en cours de traitement.

\section{Les leçons à tirer:}

- les logiciels « maison » sont potentiellement dangereux en dehors des mains de ceux qui les ont créés. Ces logiciels, développés dans le cadre des grands centres de radiothérapie pionniers de la recherche qui ont contribué à la mise en place de nouvelles méthodes de radiothérapie, trouvaient leur justification dans ce contexte. Les chaînes de traitement de radiothérapie et l'informatique associée sont devenues aujourd' hui beaucoup plus complexes et les logiciels « maison » ne se justifient plus du fait d'une insuffisance d'assurance de qualité ;

- la dosimétrie in vivo lorsqu'elle est utilisable ou une méthode dosimétrique équivalente doivent pouvoir apporter un contrôle que la dose délivrée est bien celle qui a été calculée. En conséquence, toute anomalie de concordance de résultat doit être explorée et expliquée totalement et non pas banalisée.

\section{Quatrième accident d'Épinal : 1993}

L'examen systématique de dossiers a permis de mettre en évidence une erreur de calcul pour des filtres en coins lors de radiothérapies de cancers du sein en 1993. Cette erreur est survenue lors de l'utilisation d'un nouvel accélérateur linéaire chez les 8 premières patientes traités pour un cancer du sein. Les doses délivrées ont été de 60 à 84 Gy au lieu de 50 Gy.

\section{Cinquième accident d'Épinal : 1999}

Enfin, chez 37 patientes traitées pour cancer du sein en 1999, une utilisation inappropriée d'une technique de radiothérapie a conduit à une surexposition du cœur à des doses excessives. Des complications cardiaques graves ont été observées chez 9 des patientes $(24 \%)$.

\section{Les autres événements de radiothérapie en France}

\section{L'accident de radiothérapie de Toulouse (avril 2006-avril 2007)}

L'accident de radiothérapie de Toulouse mérite une mention particulière car il a conduit à la sur irradiation de 145 personnes lors de traitements de radiochirurgie 
stéréotaxique. L'erreur a consisté en la mauvaise calibration de l'intensité du faisceau de l'appareil Novalis (accélérateur robotisé) du fait de l'utilisation d'une chambre d'ionisation de trop grande dimension par rapport à la petite taille du faisceau.

Cette erreur de calibration a été effectuée lors de la mise en route de l'appareillage en avril 2006. L'alerte a été donnée par le constructeur qui avait observé par hasard, un an après l'installation de l'appareillage, que les paramètres de calibration de l'appareillage de Toulouse qu'il s'était procuré différaient fortement des paramètres des autres installations du même type.

Le suivi des patients n'a pas généré d'alerte alors même que certains d'entre eux avaient reçu 3 fois la dose prescrite et que le taux de paralysie faciale chez les patients traités pour un neurinome de l'acoustique est in fine de l'ordre de $40 \%$.

Les leçons à tirer :

- l'installation d'un centre de radiochirurgie isolé en dehors d'une structure de radiothérapie ou en l'absence du support d'un centre de radiothérapie est à rejeter ;

- la formation initiale et continue des personnes spécialisées en radiophysique médicale, des dosimétristes et des manipulateurs est à considérer pour chaque installation de radiothérapie.

- l'installation de nouveaux appareillages de radiothérapie (nouveaux accélérateurs, robots...) doit faire l'objet d'une procédure dédiée incluant formation des personnels, élaboration de protocoles, assurance qualité...

- l'implication forte des fabricants dans la calibration des appareillages de radiochirurgie est à considérer. En effet, il y a actuellement 3 types de modalités d'installation des appareillages disponibles : le Gammaknife (appareillage composé d'environ 200 sources de ${ }^{60} \mathrm{Co}$ ) est totalement calibré par le fabricant en usine, le Cyberknife (accélérateur robotisé) est calibré sur place par les opérateurs avec contrôle immédiat du fabricant, alors que la calibration du Novalis est entièrement dans les mains des opérateurs sans contrôle particulier du fabricant.

- l'assurance de qualité lors de la recette d'installation de tout nouvel appareillage doit faire l'objet d'une attention toute particulière.

\section{Les autres événements de radiothérapie survenus en France ces dernières années}

Pour les autres événements de radiothérapie survenus en France ces dernières années, on peut identifier les problèmes significatifs suivants :

- mauvaise interface machine/logiciel. Un accident avec conséquence grave pour un seul patient (intervention chirurgicale mutilante) a résulté en 2005 de 
l'irradiation en grand champ avec un appareillage a priori capable de réaliser un champ de très petite taille pour lequel une escalade de dose était programmée. Le logiciel standard n'étant pas capable de commander une telle configuration, un logiciel issu d'un grand centre de recherche et maintes fois éprouvé a été utilisé. En revanche, la configuration particulière matériel/logiciel n'avait pas été testée. L'accident a résulté de la non commande de la fermeture du champ d'irradiation à la petite taille souhaitée, l'instruction logicielle n'ayant pas été exécutée ;

- mauvaise interface humaine. Un accident grave ayant contribué au décès d'un patient a résulté de l'irradiation en 2004 en très grand champ au lieu d'un petit champ du fait de la non prise en compte de l'unité dimensionnelle du champ (confusion entre $\mathrm{cm}$ et $\mathrm{mm}$ ) lors de la transmission orale de la demande entre 2 opérateurs. Lors de cette irradiation en mode stéréotaxique, la tête et le cou du patient avaient été exposés au lieu de l'anomalie vasculaire cérébrale. Il en était résulté en 2006 une fistule oesophago-trachéale qui avait nécessité une intervention chirurgicale lourde au décours de laquelle le patient était décédé ;

- la survenue d'une myélite radique est toujours redoutée de la part des radiothérapeutes. Un tel accident est survenu en 2007 lors de la réalisation d'une irradiation médiastinale combinée à une irradiation des chaînes cervicales. La non superposition des champs avait bien été scrupuleusement vérifiée de face mais non de profil. Il s'agit là d'une erreur de vérification, le paramétrage des 2 irradiations ayant été réalisé à un jour d'intervalle du fait d'un phénomène intercurrent ayant entraîné un retard ;

- des erreurs d'identification de patients sont fréquentes et restent fréquentes. Il s'agit d'erreur d'homonymie sur la personne physique mais aussi sur le fichier informatique associé ;

- des pertes de contrôle de sources de curiethérapie conduisent à retrouver de temps à autre des fils radioactifs dans les poubelles.

Les leçons à tirer :

- les interfaces humaines ou hommes/machines ou machines/logiciel sont sources de nombreuses erreurs possibles du fait de la multiplicité des relations et des situations. Il convient donc de faire une analyse précise de toutes les situations d'interfaces et les considérer comme situations à risque potentiel ;

- la transmission orale et la transmission papier d'instructions doivent faire l'objet d'une assurance de qualité. Au mieux, ces types de transmission ne devraient plus exister et la chaîne de réalisation devrait être intégrée par un seul constructeur;

- la déclaration des événements indésirables, la mise en place d'un registre des événements bénins et d'une cellule de retour d'expérience dans chaque service 
doivent susciter les professionnels à porter une plus grande attention aux phénomènes précurseurs qui doivent être identifiés et analysés avec rigueur ;

- des procédures doivent être mises en place à tous les niveaux et respectées afin d'éviter les erreurs d'identification des patients, la mauvaise comptabilité des sources, etc.

\section{Perspectives}

Comme on a pu le voir, les erreurs en radiothérapie sont en grande majorité du domaine du facteur organisationnel et humain et du domaine de l'assurance qualité. Dans ses contrôles, l'ASN leur porte une attention toute particulière en complément du respect de la réglementation qui est conçue pour minimiser les risques en encadrant les pratiques.

Les lettres de suite des inspections de radiothérapie viennent faire un bilan rigoureux de l'état des services et demander aux professionnels des mesures correctives à visée préventive. La publication sur le site web de l'ASN de ces lettres de suite relève de la transparence qui est demandé à l'ASN par la loi Transparence et sécurité en matière nucléaire (TSN) qui l'a mise en place.

En cas d'événement indésirable de radiothérapie, la déclaration à l'ASN est destinée à renforcer la prise de conscience des professionnels concernés et à pouvoir en informer les autres. L'information des professionnels, du public et des patients de la survenue de ces événements selon les modalités de l'échelle de communication ASN/SFRO y contribue également. L'ASN a également saisi ces dernières années les professionnels par des courriers de recommandations et des lettres circulaires destinées à faire le point sur des sujets particuliers ( $c f$. Annexe).

La communauté de radiothérapie française s'est engagée de façon responsable dans une démarche de prévention des risques depuis quelques années. On peut d'ores et déjà constater qu'elle en sort grandie même si tous les services ne sont pas encore au même niveau de progrès. On peut ainsi espérer que la France ne connaîtra plus de grands accidents de radiothérapie du type de celui d'Épinal et que la radiothérapie restera considérée par tous comme une excellente méthode de traitement des cancers.

\section{RÉFÉRENCES}

Peiffert D., Simon J.M., Eschwege F. (2007) L'accident d'Épinal : passé, présent, avenir, Cancer Radiothérapie 11, 309-312.

Marchesi V., Aigle D., Peiffert D., Noel A., Simon J.M. (2009) Sécurisation de l'activité de radiothérapie bi-site dans le cadre de la reprise des traitements au centre hospitalier d'Épinal par l'équipe du centre Alexis-Vautrin de Nancy, Cancer Radiothérapie, DOI: 10.1016/j.canrad.2009.05.011. 


\section{Annexe : Chronologie des actions de l'ASN en radiothérapie}

Août 2004 Loi 2004-806 du 9 août 2004 relative à la politique de santé publique créant une inspection de la radioprotection.

Avril 2005 Déclaration un incident de radiothérapie au CHU de Grenoble. Diffusion à tous les services de radiothérapie d'une lettre circulaire.

DGSNR/Afssaps sur la radioprotection des patients en radiothérapie.

Juin 2005 Diffusion de l'ordre du jour type pour la visite d'une unité de radiothérapie comprenant certains aspects relatifs à la radioprotection des patients.

Février 2006 Déclaration d'un accident de radiothérapie au CH de Lyon-Sud.

Avril 2006 Envoi d'une circulaire par la DGSNR à tous les centres de radiothérapie sur la prise en compte des Facteurs organisationnels et humains $(\mathrm{FOH})$ en radiothérapie.

Mai 2006 Visite du service de radiothérapie du CH J. Monnet d'Épinal par la division de Strasbourg de la DGSNR et demande d'information sur la mise en œuvre de la circulaire DGSNR d'avril 2006 relative aux FOH.

Juin 2006 Décret $N^{\circ}$ 2006-694 du 13 juin 2006 fixant les modalités de désignation, d'habilitation et de prestation de serment des inspecteurs de la radioprotection.

Juillet 2006 Information de la DHOS d'un accident de radiothérapie au CH J. Monnet d'Épinal.

Inspection réactive ASN au $\mathrm{CH}$ J. Monnet d'Épinal en liaison avec l'ARH et l'IRSN.

Envoi de la lettre de suite d'inspection au CH. J. Monnet demandant des actions correctives et compléments d'information.

Septembre Création par l'ASN d'un groupe de travail associant les 2006 administrations sanitaires et les professionnels avec l'appui de l'IRSN en vue d'élaborer des recommandations concernant la sécurité des traitements de radiothérapie.

Arrêté du 13 septembre 2006 désignant 62 premiers inspecteurs de la radioprotection de la DGSNR.

Octobre 2006 Demande de mission conjointe IGAS-ASN sur l'accident d'Épinal.

Saisine de l'IRSN par le ministre chargé de la santé (radiopathologie). 
Communiqué de l'ASN annonçant qu'elle va :

- renforcer ses inspections,

- procéder à la vérification des actions engagées par les établissements hospitaliers en matière de formation,

- diffuser un guide de déclaration des événements, approfondir en liaison avec l'Afssaps,

- les conditions d'utilisation des logiciels et leur ergonomie.

Décembre Instruction aux divisions de l'ASN relative à l'enquête pour 2006 évaluer les moyens dédiés à la radiophysique médicale dans tous les services de radiothérapie.

Réception de la liste des autres centres de radiothérapie utilisant le logiciel de planification des traitements CADPLAN transmise par l'Afssaps.

Instruction aux divisions de l'ASN de vérifier l'absence d'erreur de paramétrage dans les autres centres de radiothérapie communiqués par l'Afssaps utilisant le logiciel de traitement et de planification des traitements CADPLAN.

Transmission par l'IGAS à la ministre d'un rapport d'étape mentionnant de «nombreuses vérifications en cours», le « manque encore d'éléments d'informations sur certains points et une analyse trop précoce des responsabilités risquant de compromettre cette recherche d'informations ».

Janvier 2007 Mobilisation et responsabilisation des professionnels, les invitant à faire part de leurs propositions pour améliorer la sécurité des traitements en radiothérapie (courriers à la SFPM et à la SFRO).

Février 2007 Remise du rapport IGAS/ASN sur l'accident d'Épinal (cohorte des 24 patients).

Mars 2007 Séminaire de formation des inspecteurs de la radioprotection sur la radiothérapie.

Remise du rapport de mission IRSN sur l'évaluation des pratiques de radiothérapie au CH J. Monnet d'Épinal, période 2001-2006. Mise en évidence d'une cohorte de 397 autres personnes concernées par des erreurs de comptage de dose.

Avril 200761 visites en radiothérapie organisées en 2006 (Rapport annuel sur la sûreté nucléaire et la radioprotection en 2006).

Publication du résultat de l'enquêté sur la radiophysique médicale (Rapport annuel sur la sûreté nucléaire et la radioprotection en 2006).

Diffusion aux inspecteurs de la radioprotection du canevas d'inspection - thème prioritaire 2007 « Radioprotection des 
patients en radiothérapie externe : prévention des incidents par une approche sur les facteurs organisationnels et humaines ».

Mai 2007 Déclaration à l'ASN de l'accident de radiothérapie stéréotaxique au CHU.

Hopital Rangueil de Toulouse.

Inspection réactive par la division de Bordeaux de l'ASN au CHU Hôpital.

Rangueil de Toulouse et publication du rapport d'inspection sur le site internet de l'ASN.

Diffusion à tous les centres de radiothérapie d'une lettre circulaire relative aux bonnes pratiques pour l'utilisation de l'imagerie portale en radiothérapie.

Diffusion à tous les centres de radiothérapie d'une lettre circulaire relative à la calibration des faisceaux.

Juin 2007 Diffusion d'un guide de déclaration des événements significatifs de radioprotection, pour expérimentation.

Juin 2007 Lettre de mission du ministre chargée de la santé en vue de mener des investigations complémentaires en coordination avec l'IGAS sur l'accident de radiothérapie au CHU hôpital Rangueil de Toulouse.

Juin 2007 Suspension temporaire des autorisations de quatre centres de radiothérapie stéréotaxique utilisant un cadre de stéréotaxie Brainlab.

Juillet 2007 Publication d'une échelle ASN/SFRO de classement de la sévérité des événements de radiothérapie, en vue d'une expérimentation.

Août 2007 Publication par l'Afssaps en liaison avec l'ASN de recommandations aux fabricants relatives aux dispositifs médicaux de radiothérapie.

Novembre Publication de la feuille de route des mesures nationales pour la radiothérapie.

Participation de l'ASN à l'audition publique de l'OPECST sur la radiothérapie.

Publication du guide de radiothérapie des tumeurs par la SFRO en coordination avec l'Afssaps.

Décembre Remise du rapport d'expertise IRSN sur l'évaluation des 2007 pratiques de radiothérapie au CH J. Monnet d'Épinal, période 1987-2000. Mise en évidence d'une erreur systématique de calcul de temps de traitement affectant à des degrés divers 4500 patients. 
Janvier 2008 Rapport sur l'enquête ASN/IGAS sur l'accident de radiothérapie de Toulouse.

Février/ Enquête conjointe ASN/Afssaps auprès des centres de Mars 2008 radiothérapie en France afin de mesurer la place occupée aujourd'hui par les logiciels non commerciaux développés en interne par les établissements.

Avril 2008 Publication de la synthèse des inspections réalisées dans tous les centres de radiothérapie en 2007.

Mai-Juin 2008 Inspection des 27 centres identifiés parmi les centres les plus vulnérables en 2007 et réinspection de l'ensemble des centres de radiothérapie.

Juin 2008 Publication par l'INCa de l'Observatoire national de la radiothérapie en coordination avec l'ASN.

Juillet 2008 Révision de l'échelle ASN/SFRO.

Publication sur le site Internet de l'ASN des lettres de suite des inspections réalisées depuis le $1^{\text {er }}$ janvier 2008.

Décision $\mathrm{N}^{\circ}$ 2008-DC-0103 de l'Autorité de sûreté nucléaire du $1^{\mathrm{er}}$ juillet 2008 fixant les obligations d'assurance de la qualité en radiothérapie définies à l'article R. 1333-59 du code de la santé publique.

Publication de recommandations aux services de radiothérapie par l'Afssaps à la suite de l'enquête réalisée, de février à mars 2008, sur les logiciels non commerciaux développés en interne par les établissements.

Décembre Installation du comité national de suivi des mesures nationales 2008 pour la radiothérapie.

Rapport de la Commission des suites de l'IGAS concernant l'accident de radiothérapie d'Épinal.

Janvier 2009 Suspension de l'autorisation du centre de radiothérapie de Blois.

Février 2009 Suspension de l'autorisation du centre de radiothérapie de Gap. Suspension de l'autorisation de centre de radiothérapie de Roanne.

Mars 2009 Homologation de la décision no 2008-DC-0103 de l'Autorité de sûreté nucléaire du $1^{\text {er }}$ juillet 2008 fixant les obligations d'assurance de la qualité en radiothérapie définies à l'article R. 1333-59 du code de la santé publique (JORF du 25 mars).

Publication du guide d'inspection radiothérapie relatif aux thèmes d'inspection prioritaires 2009.

Avril 2009 Levée de la suspension de l'autorisation du centre de radiothérapie de Blois. 